\title{
Log-Convexity Properties of Schur Functions and Generalized Hypergeometric Functions of Matrix Argument
}

\author{
Donald St. P. Richards*
}

August 22, 2009

\begin{abstract}
We establish a positivity property for the difference of products of certain Schur functions, $s_{\lambda}(x)$, where $\lambda$ varies over a fundamental Weyl chamber in $\mathbb{R}^{n}$ and $x$ belongs to the positive orthant in $\mathbb{R}^{n}$. Further, we generalize that result to the difference of certain products of arbitrary numbers of Schur functions. We also derive a log-convexity property of the generalized hypergeometric functions of two Hermitian matrix arguments, and we show how that result may be extended to derive higher-order log-convexity properties.
\end{abstract}

\section{Introduction}

In this paper, we derive some positivity properties of the Schur functions and the generalized hypergeometric functions of matrix argument.

The Schur functions, $s_{\lambda}$, are indexed by vectors $\lambda \in \mathbb{R}^{n}$. For the case in which these vectors are partitions, the functions $s_{\lambda}$ are polynomials and collectively form a linear basis of $\Lambda$, the ring of symmetric functions in the variables $x_{1}, x_{2}, \ldots \in \mathbb{R}$. The functions $s_{\lambda}$ are ubiquitous, arising in the algebra of symmetric functions [15]; in combinatorics, in the theory of semi-standard Young tableaux [18; and in the representation theory of $\operatorname{GL}(n, \mathbb{C})$, the complex general linear group [7, 15]. In this paper, we will make use of the interpretation in mathematical statistics and in the theory of total positivity of the Schur functions $s_{\lambda}$ as the "complex zonal polynomials" [5, 9, or as spherical functions in harmonic analysis on spaces of positive definite Hermitian matrices [5, 7].

For $\lambda=\left(\lambda_{1}, \ldots, \lambda_{n}\right)$ and $\mu=\left(\mu_{1}, \ldots, \mu_{n}\right)$ in $\mathbb{R}^{n}$ define the least upper bound,

$$
\lambda \vee \mu:=\left(\max \left(\lambda_{1}, \mu_{1}\right), \ldots, \max \left(\lambda_{n}, \mu_{n}\right)\right),
$$

${ }^{*}$ Department of Statistics, Penn State University, University Park, PA 16802, USA.

${ }^{\star}$ Supported in part by National Science Foundation grants DMS-0705210.

2000 Mathematics Subject Classification: Primary 05E05, 33C67; Secondary 05A17, 15A15, 60E15.

Key words and phrases. Finite distributive lattice; FKG inequality; generalized hypergeometric function of matrix argument; log-supermodular; monomial-positivity; partition; multivariate total positivity; Schur function; Schur-positivity; Sylvester's formula; symmetric function; Young tableaux; zonal polynomial. 
and the greatest lower bound,

$$
\lambda \wedge \mu:=\left(\min \left(\lambda_{1}, \mu_{1}\right), \ldots, \min \left(\lambda_{n}, \mu_{n}\right)\right) .
$$

These operations induce on $\mathbb{R}^{n}$ a partial ordering, the lexicograpghic ordering, and $\mathbb{R}^{n}$ then becomes a distributive lattice.

For $x=\left(x_{1}, \ldots, x_{n}\right) \in \mathbb{C}^{n}$, define

$$
\Delta_{\lambda}(x)=\operatorname{det}\left(x_{i}^{\lambda_{j}+n-j}\right)
$$

the $n \times n$ determinant with $(i, j)$ th entry $x_{i}^{\lambda_{j}+n-j}$. For the case in which $\lambda=0$, the zero vector, it is well-known that (1.3) reduces to the Vandermonde determinant,

$$
\Delta_{0}(x):=\operatorname{det}\left(x_{i}^{n-j}\right)=\prod_{i<j}\left(x_{i}-x_{j}\right)
$$

The Schur function indexed by the vector $\lambda$ is defined as

$$
s_{\lambda}(x)=\frac{\Delta_{\lambda}(x)}{\Delta_{0}(x)} \equiv \frac{\operatorname{det}\left(x_{i}^{\lambda_{j}+n-j}\right)}{\prod_{i<j}\left(x_{i}-x_{j}\right)},
$$

with the convention that L'Hospital's formula is applied whenever there are equalities among $x_{1}, \ldots, x_{n}$. For the case in which $\lambda$ is a partition, it is well-known that this definition of $s_{\lambda}$ is equivalent to the combinatorial definition in terms of Young tableaux.

Let $\mathcal{W}=\left\{\left(\lambda_{1}, \ldots, \lambda_{n}\right) \in \mathbb{R}^{n}: \lambda_{1} \geq \cdots \geq \lambda_{n}\right\}$, a fundamental Weyl chamber in $\mathbb{R}^{n}$, and denote by $\mathbb{R}_{+}$the positive real line. Then our first aim is to establish by analytical methods the following log-convexity property of the Schur functions.

Theorem 1.1. Let $\lambda, \mu \in \mathcal{W}$ and $x \in \mathbb{R}_{+}^{n}$. Then, $s_{\lambda \vee \mu}(x) s_{\lambda \wedge \mu}(x)-s_{\lambda}(x) s_{\mu}(x) \geq 0$.

As a consequence of this result, we will also derive in Theorem 2.1] an extension that involves any finite number of vectors drawn from $\mathcal{W}$.

A symmetric function $p$ in the variables $x_{1}, \ldots, x_{n} \in \mathbb{R}$ is called monomial-positive if, on expressing $p$ as a linear combination of monomials in $x_{1}, \ldots, x_{n}$, each monomial term has a nonnegative coefficient. Similarly, $p$ is Schur-positive if in the expansion of $p$ as a linear combination of Schur functions each term has a nonnegative coefficient.

Recall that $\lambda \in \mathcal{W}$ is a partition if $\lambda_{1}, \ldots, \lambda_{n}$ are nonnegative integers. Corresponding to each partition $\lambda$ is a unique Young diagram [15, 18. In particular, given partitions $\lambda$ and $\mu$, the partitions corresponding to the union and intersection, respectively, of the Young diagrams of $\lambda$ and $\mu$ are given by (1.1) and (1.2).

In some recent articles Lam, et al. [12, 13] have established some interesting positivity properties of the Schur functions. A consequence of the work of Lam, et al. [12, 13] is the following result.

Theorem 1.2. (Lam, et al. 12, 13]) For partitions $\lambda$ and $\mu$, the symmetric function $s_{\lambda \vee \mu} s_{\lambda \wedge \mu}-s_{\lambda} s_{\mu}$ is monomial-positive and Schur-positive. 
For $x_{1}, \ldots, x_{n} \in \mathbb{R}_{+}$, the positive real line, it is well-known that $s_{\lambda}\left(x_{1}, \ldots, x_{n}\right) \geq 0$, so Theorem 1.2 implies Theorem 1.1 for the case in which $\lambda$ and $\mu$ are partitions. Nevertheless, the two results do not seem to be directly comparable, for Theorem 1.1] applies to all $\lambda, \mu \in \mathcal{W}$, but proves positivity only, whereas Theorem 1.2 applies to partitions $\lambda, \mu$ only, but proves monomial- and Schur-positivity. Moreover, the markedly different methods by which the two are established further underscores their incomparability.

The second aim of this paper is to derive log-convexity properties of the (generalized) hypergeometric functions of two Hermitian matrix arguments [5, 9]. These functions are infinite series of "complex" zonal polynomials with coefficients extending the classical rising factorial, and they generalize the classical hypergeometric functions in many ways. For suitably chosen scalars $a_{1}, \ldots, a_{p}, b_{1}, \ldots, b_{q} \in \mathbb{C}$, denote by

$$
{ }_{p} F_{q}\left(\begin{array}{l}
a_{1}, \ldots, a_{p} \\
b_{1}, \ldots, b_{q}
\end{array} ; x, y\right)
$$

the hypergeometric function of $n \times n$ Hermitian matrix arguments, $x$ and $y$. By applying properties of the Schur functions established in Section 2 together with application of the FKG inequality [4, 16], we shall establish the positivity of certain differences of products of these hypergeometric functions. By means of the generalized FKG inequalities in [16], we obtain results in which these products involve as many as five ${ }_{p} F_{q}$ functions of two Hermitian matrix arguments.

\section{Log-convexity properties of the Schur functions}

A function $\phi: \mathbb{R}^{n} \rightarrow[0, \infty)$ is called multivariate totally positive of order $2\left(\mathrm{MTP}_{2}\right)$ if

$$
\phi(\lambda \vee \mu) \phi(\lambda \wedge \mu) \geq \phi(\lambda) \phi(\mu)
$$

for all $\lambda, \mu \in \mathbb{R}^{n}$. We remark that the " $\mathrm{MTP}_{2}$ " terminology is common in mathematical statistics and parts of probability theory [11, 16]; in combinatorics [1, p. 83], game theory, and economics [19, p. 64], the $\mathrm{MTP}_{2}$ concept is known as log-supermodularity; and in mathematical physics and related areas of probability [2, 4, 8, it is known as the FKG condition.

There is an analytical characterization of the class of positive, sufficiently smooth $\mathrm{MTP}_{2}$ functions [2, 8, 11, 14]: Suppose that $\phi: \mathbb{R}^{n} \rightarrow \mathbb{R}_{+}$is in $C^{2}\left(\mathbb{R}^{n}\right)$; then $\phi$ is $\mathrm{MTP}_{2}$ if and only if

$$
\frac{\partial^{2}}{\partial \lambda_{i} \partial \lambda_{j}} \log \phi(\lambda) \geq 0
$$

for all $1 \leq i \neq j \leq n$.

As an application of this characterization, it follows that if $\phi_{1}, \phi_{2}: \mathbb{R}^{n} \rightarrow \mathbb{R}_{+}$both are in $C^{2}\left(\mathbb{R}^{n}\right)$ and are $\mathrm{MTP}_{2}$ then the product $\phi_{1} \phi_{2}$ also is $\mathrm{MTP}_{2}$. This result holds, more generally, for all $\mathrm{MTP}_{2}$ functions, a result which is established by Karlin and Rinott [1] by algebraic methods.

By means of the characterization (2.2), we now establish Theorem 1.1 via analytical methods; in so doing, we write $s_{\lambda}(x)$ simply as $s_{\lambda}$. 
Proof of Theorem 1.1. To ascertain the sign of $\partial^{2} \log s_{\lambda} / \partial \lambda_{i} \partial \lambda_{j}$ for any $i \neq j$, it suffices to take $(i, j)=(1,2)$, for all other cases are resolved in the same way. Let $\alpha_{j}=\lambda_{j}+n-j$, $1 \leq j \leq n$, so that $\alpha_{1}>\cdots>\alpha_{n}$, set $\alpha=\left(\alpha_{1}, \ldots, \alpha_{n}\right)$, and define

$$
\widetilde{\Delta}_{\alpha}(x)=\operatorname{det}\left(x_{i}^{\alpha_{j}}\right) .
$$

By (1.3) -(1.5), we have

$$
\begin{aligned}
\Delta_{\lambda}^{2} \cdot \frac{\partial^{2}}{\partial \lambda_{1} \lambda_{2}} \log s_{\lambda} & =\Delta_{\lambda} \cdot \frac{\partial^{2}}{\partial \lambda_{1} \lambda_{2}} \Delta_{\lambda}-\frac{\partial}{\partial \lambda_{1}} \Delta_{\lambda} \cdot \frac{\partial}{\partial \lambda_{2}} \Delta_{\lambda} \\
& =\widetilde{\Delta}_{\alpha} \cdot \frac{\partial^{2}}{\partial \alpha_{1} \alpha_{2}} \widetilde{\Delta}_{\alpha}-\frac{\partial}{\partial \alpha_{1}} \widetilde{\Delta}_{\alpha} \cdot \frac{\partial}{\partial \alpha_{2}} \widetilde{\Delta}_{\alpha}
\end{aligned}
$$

To simplify this difference of product terms, we proceed as in [17, Section 3], applying a result derived by Karlin [10, p. 7, eq. (0.16)] from Sylvester's formula for compound determinants: For column vectors $\boldsymbol{a}, \boldsymbol{b}, \boldsymbol{f}_{1}, \ldots, \boldsymbol{f}_{n-2} \in \mathbb{R}^{n}$, define the determinant

$$
D\left(\boldsymbol{a}, \boldsymbol{b}, \boldsymbol{f}_{1}, \ldots, \boldsymbol{f}_{n-2}\right)=\operatorname{det}\left(\boldsymbol{a}, \boldsymbol{b}, \boldsymbol{f}_{1}, \ldots, \boldsymbol{f}_{n-2}\right) .
$$

Then, for $\boldsymbol{a}_{1}, \boldsymbol{a}_{2}, \boldsymbol{b}_{1}, \boldsymbol{b}_{2} \in \mathbb{R}^{n}$,

$$
\begin{array}{ll}
\left|\begin{array}{ll}
D\left(\boldsymbol{a}_{1}, \boldsymbol{b}_{1}, \boldsymbol{f}_{1}, \ldots, \boldsymbol{f}_{n-2}\right) & D\left(\boldsymbol{a}_{1}, \boldsymbol{b}_{2}, \boldsymbol{f}_{1}, \ldots, \boldsymbol{f}_{n-2}\right) \\
D\left(\boldsymbol{a}_{2}, \boldsymbol{b}_{1}, \boldsymbol{f}_{1}, \ldots, \boldsymbol{f}_{n-2}\right) & D\left(\boldsymbol{a}_{2}, \boldsymbol{b}_{2}, \boldsymbol{f}_{1}, \ldots, \boldsymbol{f}_{n-2}\right)
\end{array}\right| \\
\quad=D\left(\boldsymbol{a}_{1}, \boldsymbol{a}_{2}, \boldsymbol{f}_{1}, \ldots, \boldsymbol{f}_{n-2}\right) \cdot D\left(\boldsymbol{b}_{1}, \boldsymbol{b}_{2}, \boldsymbol{f}_{1}, \ldots, \boldsymbol{f}_{n-2}\right) .
\end{array}
$$

For $u \in \mathbb{R}_{+}$and $v \in \mathbb{R}$, define the kernel $K: \mathbb{R}_{+} \times \mathbb{R} \rightarrow \mathbb{R}_{+}$by

$$
K(u, v)=u^{v}
$$

set

$$
\begin{gathered}
\boldsymbol{a}_{1}=\left(\begin{array}{c}
K\left(x_{1}, \alpha_{1}\right) \\
\vdots \\
K\left(x_{n}, \alpha_{1}\right)
\end{array}\right), \quad \boldsymbol{a}_{2}=\frac{\partial}{\partial \alpha_{1}}\left(\begin{array}{c}
K\left(x_{1}, \alpha_{1}\right) \\
\vdots \\
K\left(x_{n}, \alpha_{1}\right)
\end{array}\right), \\
\boldsymbol{b}_{1}=\left(\begin{array}{c}
K\left(x_{1}, \alpha_{2}\right) \\
\vdots \\
K\left(x_{n}, \alpha_{2}\right)
\end{array}\right), \quad \boldsymbol{b}_{2}=\frac{\partial}{\partial \alpha_{2}}\left(\begin{array}{c}
K\left(x_{1}, \alpha_{2}\right) \\
\vdots \\
K\left(x_{n}, \alpha_{2}\right)
\end{array}\right) ;
\end{gathered}
$$

and, for $j=1, \ldots, n-2$, set

$$
\boldsymbol{f}_{j}=\left(\begin{array}{c}
K\left(x_{1}, \alpha_{j}\right) \\
\vdots \\
K\left(x_{n}, \alpha_{j}\right)
\end{array}\right) \text {. }
$$

Then

$$
D\left(\boldsymbol{a}_{1}, \boldsymbol{b}_{1}, \boldsymbol{f}_{1}, \ldots, \boldsymbol{f}_{n-2}\right)=\widetilde{\Delta}_{\alpha}(x),
$$




$$
\begin{aligned}
& D\left(\boldsymbol{a}_{1}, \boldsymbol{b}_{2}, \boldsymbol{f}_{1}, \ldots, \boldsymbol{f}_{n-2}\right)=\frac{\partial}{\partial \alpha_{2}} \widetilde{\Delta}_{\alpha}(x), \\
& D\left(\boldsymbol{a}_{2}, \boldsymbol{b}_{1}, \boldsymbol{f}_{1}, \ldots, \boldsymbol{f}_{n-2}\right)=\frac{\partial}{\partial \alpha_{1}} \widetilde{\Delta}_{\alpha}(x),
\end{aligned}
$$

and

$$
D\left(\boldsymbol{a}_{2}, \boldsymbol{b}_{2}, \boldsymbol{f}_{1}, \ldots, \boldsymbol{f}_{n-2}\right)=\frac{\partial^{2}}{\partial \alpha_{1} \partial \alpha_{2}} \widetilde{\Delta}_{\alpha}(x) .
$$

Substituting these results into (2.3) and (2.4), we obtain

$$
\begin{aligned}
\Delta_{\lambda}^{2} \cdot \frac{\partial^{2}}{\partial \lambda_{1} \lambda_{2}} \log s_{\lambda} & =\widetilde{\Delta}_{\alpha} \cdot \frac{\partial^{2}}{\partial \alpha_{1} \alpha_{2}} \widetilde{\Delta}_{\alpha}-\frac{\partial}{\partial \alpha_{1}} \widetilde{\Delta}_{\alpha} \cdot \frac{\partial}{\partial \alpha_{2}} \widetilde{\Delta}_{\alpha} \\
& =\left|\begin{array}{ll}
D\left(\boldsymbol{a}_{1}, \boldsymbol{b}_{1}, \boldsymbol{f}_{1}, \ldots, \boldsymbol{f}_{n-2}\right) & D\left(\boldsymbol{a}_{1}, \boldsymbol{b}_{2}, \boldsymbol{f}_{1}, \ldots, \boldsymbol{f}_{n-2}\right) \\
D\left(\boldsymbol{a}_{2}, \boldsymbol{b}_{1}, \boldsymbol{f}_{1}, \ldots, \boldsymbol{f}_{n-2}\right) & D\left(\boldsymbol{a}_{2}, \boldsymbol{b}_{2}, \boldsymbol{f}_{1}, \ldots, \boldsymbol{f}_{n-2}\right)
\end{array}\right| \\
& =D\left(\boldsymbol{a}_{1}, \boldsymbol{a}_{2}, \boldsymbol{f}_{1}, \ldots, \boldsymbol{f}_{n-2}\right) \cdot D\left(\boldsymbol{b}_{1}, \boldsymbol{b}_{2}, \boldsymbol{f}_{1}, \ldots, \boldsymbol{f}_{n-2}\right) .
\end{aligned}
$$

Next,

$$
\begin{aligned}
D\left(\boldsymbol{a}_{1}, \boldsymbol{a}_{2}, \boldsymbol{f}_{1}, \ldots, \boldsymbol{f}_{n-2}\right) & =\left|\begin{array}{ccccc}
K\left(x_{1}, \alpha_{1}\right) & \frac{\partial}{\partial \alpha_{1}} K\left(x_{1}, \alpha_{1}\right) & K\left(x_{1}, \alpha_{3}\right) & \cdots & K\left(x_{1}, \alpha_{n}\right) \\
\vdots & \vdots & \vdots & \cdots & \vdots \\
K\left(x_{n}, \alpha_{1}\right) & \frac{\partial}{\partial \alpha_{1}} K\left(x_{n}, \alpha_{1}\right) & K\left(x_{n}, \alpha_{3}\right) & \cdots & K\left(x_{n}, \alpha_{n}\right)
\end{array}\right| \\
& \equiv \lim _{\alpha_{2} \rightarrow \alpha_{1}} \frac{\operatorname{det}\left(K\left(x_{i}, \alpha_{j}\right)\right)}{\alpha_{2}-\alpha_{1}} .
\end{aligned}
$$

It is well-known [10] that the kernel $K$ in (2.5) is strictly totally positive of order infinity $\left(\mathrm{STP}_{\infty}\right)$, i.e. $\operatorname{det}\left(K\left(x_{i}, \alpha_{j}\right)\right)>0$ for all $x_{1}>\cdots>x_{n}>0, \alpha_{1}>\cdots>\alpha_{n}$, and all $n \geq 1$. Hence, in the above limit, the numerator is positive and the denominator is negative; therefore, $D\left(\boldsymbol{a}_{1}, \boldsymbol{a}_{2}, \boldsymbol{f}_{1}, \ldots, \boldsymbol{f}_{n-2}\right) \leq 0$. (We remark that the limit can be shown to be negative by utilizing the fact [10, pp. 16] that the kernel $K$ also is extended totally positive of order infinity $\left(\mathrm{ETP}_{\infty}\right)$; however, this result is not needed for our purposes.)

Similarly, we deduce that $D\left(\boldsymbol{b}_{1}, \boldsymbol{b}_{2}, \boldsymbol{f}_{1}, \ldots, \boldsymbol{f}_{n-2}\right) \leq 0$, and then it follows from (2.6) that for $\lambda \in \mathcal{W}$ and $x_{1}>\cdots>x_{n}>0$,

$$
\frac{\partial^{2}}{\partial \lambda_{i} \partial \lambda_{j}} \log s_{\lambda}(x)>0,
$$

$i \neq j$. By the characterization (2.2) of functions that are positive, $\mathrm{MTP}_{2}$, and $C^{2}$, we deduce that if $\lambda, \mu \in \mathcal{W}$ and $x_{1}>\cdots>x_{n}>0$ then

$$
s_{\lambda \vee \mu}(x) s_{\lambda \wedge \mu}(x)-s_{\lambda}(x) s_{\mu}(x) \geq 0 .
$$

As a limiting case, we find that positivity also holds if $x_{1} \geq \cdots \geq x_{n}$; and because this function is symmetric in $x_{1}, \ldots, x_{n}$, the condition $x_{1} \geq \cdots \geq x_{n}$ can be dispensed with. Consequently, (2.7) holds for all $x \in \mathbb{R}_{+}^{n}$.

Extending Theorem 1.1, we have the following result. 
Theorem 2.1. Let $p$ be a positive integer and, for $\kappa_{1}, \ldots, \kappa_{p} \in \mathcal{W}$, define

$$
\nu_{j}=\bigvee_{1 \leq i_{1}<\cdots<i_{j} \leq p}\left(\kappa_{i_{1}} \wedge \cdots \wedge \kappa_{i_{j}}\right)
$$

$j=1, \ldots, p$. Then, for $x \in \mathbb{R}_{+}^{n}$ and any positive integer $q$, where $1 \leq q \leq p$,

$$
\prod_{j=q}^{p}\left(s_{\nu_{j}}(x)\right)^{\left(\begin{array}{c}
j-1 \\
q-1
\end{array}\right)}-\prod_{1 \leq i_{1}<\cdots<i_{q} \leq p} s_{\kappa_{i_{1}} \wedge \cdots \wedge \kappa_{i_{q}}}(x) \geq 0 .
$$

Moreover, these inequalities remain valid if the operations $\vee$ and $\wedge$ are interchanged; that is, if

$$
\widetilde{\nu}_{j}=\bigwedge_{1 \leq i_{1}<\cdots<i_{j} \leq p}\left(\kappa_{i_{1}} \vee \cdots \vee \kappa_{i_{j}}\right)
$$

$j=1, \ldots, p$, then, for $x \in \mathbb{R}_{+}^{n}$ and any positive integer $q$, where $1 \leq q \leq p$,

$$
\prod_{j=q}^{p}\left(s_{\widetilde{\nu}_{j}}(x)\right)^{\left(\begin{array}{c}
j-1 \\
q-1
\end{array}\right)}-\prod_{1 \leq i_{1}<\cdots<i_{q} \leq p} s_{\kappa_{i_{1}} \vee \cdots \vee \kappa_{i_{q}}}(x) \geq 0 .
$$

Proof. By Theorem 1.1] $s_{\lambda \vee \mu}(x) s_{\lambda \wedge \mu}(x) \geq s_{\lambda}(x) s_{\mu}(x)$; equivalently,

$$
\log s_{\lambda \vee \mu}(x)+\log s_{\lambda \wedge \mu}(x) \geq \log s_{\lambda}(x)+\log s_{\mu}(x),
$$

so the function $\lambda \mapsto-\log s_{\lambda}(x)$ is subadditive in the sense defined by Fan [3]. On applying the theorem of Fan, we obtain the inequality,

$$
\sum_{j=q}^{p}\left(\begin{array}{l}
j-1 \\
q-1
\end{array}\right) \log \left(s_{\nu_{j}}(x)\right) \geq \sum_{1 \leq i_{1}<\cdots<i_{q} \leq p} \log s_{\kappa_{i_{1}} \wedge \cdots \wedge \kappa_{i_{q}}}(x),
$$

which yields (2.8) by exponentiation.

Finally, the dual result (2.9) is obtained similarly.

The results in Theorem 2.1 also raise the combinatorial problem of whether the symmetric functions in (2.8) and (2.9) are monomial-positive or Schur-positive.

\section{Log-convexity properties of the hypergeometric functions of matrix argument}

In this section, we apply Theorem 1.1 and the FKG inequality to derive log-convexity properties of the (generalized) hypergeometric functions of two Hermitian matrix arguments.

Let $\phi: \mathbb{R}^{n} \rightarrow \mathbb{R}$ be a $\mathrm{MTP}_{2}$ probability density function; let $f: \mathbb{R}^{n} \rightarrow \mathbb{R}$; and denote by $\mathbb{E}(f)$ the expectation, whenever it exists, of $f$ with respect to the probability 
distribution corresponding to $\phi$. A function $f: \mathbb{R}^{n} \rightarrow \mathbb{R}$ is called increasing if $f(x)$ is monotone increasing in each coordinate of the argument $x \in \mathbb{R}^{n}$.

We recall the FKG inequality [4, 16]: If $f$ and $g$ are increasing functions on $\mathbb{R}^{n}$ then

$$
\mathbb{E}(f g)-\mathbb{E}(f) \mathbb{E}(g) \geq 0,
$$

Let $\lambda=\left(\lambda_{1}, \ldots, \lambda_{n}\right)$ be a partition. The length, $\ell(\lambda)$, of $\lambda$ is the largest integer $l$ such that $\lambda_{l}>0$; and the weight of $\lambda$ is $|\lambda|:=\lambda_{1}+\cdots+\lambda_{n}$. We denote by $\mathcal{P}_{n}$ the set of all partitions $\lambda$ of length $\ell(\lambda) \leq n$.

To apply the FKG inequality, we construct a $\mathrm{MTP}_{2}$ probability density function on $\mathcal{P}_{n}$, and then we deduce certain hypergeometric function inequalities by a careful choice of the functions $f$ and $g$ in (3.1).

Let $\mathcal{H}_{n}$ denote the space of $n \times n$ Hermitian matrices, and $\mathcal{H}_{n}^{+}$denote the positive definite matrices in $\mathcal{H}_{n}$. Whenever $x \in \mathcal{H}_{n}$, with eigenvalues $x_{1}, \ldots, x_{n}$, we write $s_{\lambda}(x) \equiv s_{\lambda}\left(x_{1}, \ldots, x_{n}\right)$, an interpretation that is based on the interpretation of the Schur functions as "class functions" in the representation theory of $\operatorname{GL}(n, \mathbb{C})$.

We shall construct positive coefficients $c_{\lambda}, \lambda \in \mathcal{P}_{n}$, such that $c_{\lambda}$ is $\operatorname{MTP}_{2}$ in $\lambda$; and then,for fixed $x \in \mathcal{H}_{n}^{+}$, we form the function,

$$
\phi(\lambda)=\frac{c_{\lambda} s_{\lambda}(x)}{\sum_{\mu \in \mathcal{P}_{n}} c_{\mu} s_{\mu}(x)},
$$

$\lambda \in \mathcal{P}_{n}$. Since $x \in \mathcal{H}_{n}^{+}$then $s_{\lambda}(x)>0$, and hence $\phi(\lambda)>0, \lambda \in \mathcal{P}_{n}$. Therefore, subject to the absolute convergence of the denominator in (3.2), $\phi$ is a probability density function on $\mathcal{P}_{n}$. Moreover, because the class of $\mathrm{MTP}_{2}$ functions is closed under pointwise multiplication [11, we shall deduce from the $\mathrm{MTP}_{2}$ nature of the coefficients $c_{\lambda}$ and the functions $s_{\lambda}(x)$ that $\phi$ also is $\mathrm{MTP}_{2}$.

For $a \in \mathbb{C}$ define the classical shifted factorial, $(a)_{k}=a(a+1) \cdots(a+k-1)$, $k=0,1,2, \ldots$ and, for $\lambda \in \mathcal{P}_{n}$, define the partitional shifted factorial,

$$
(a)_{\lambda}=\prod_{j=1}^{n}(a-j+1)_{\lambda_{j}} .
$$

Lemma 3.1. Let $a_{1}, \ldots, a_{p}, b_{1}, \ldots, b_{q}>n-1, y \in \mathcal{H}_{n}^{+}$, and define

$$
c_{\lambda}=\frac{\left(a_{1}\right)_{\lambda} \cdots\left(a_{p}\right)_{\lambda}}{\left(b_{1}\right)_{\lambda} \cdots\left(b_{q}\right)_{\lambda}(n)_{\lambda}} s_{\lambda}(y)
$$

$\lambda \in \mathcal{P}_{n}$. Then, $c_{\lambda}$ is $M T P_{2}$ in $\lambda$.

Proof. If $a>n-1$ then the function $\lambda \mapsto(a)_{\lambda}, \lambda \in \mathcal{P}_{n}$ clearly is positive. Moreover, $(a)_{\lambda}$ is $\mathrm{MTP}_{2}$, for it is straightforward to verify that $(a)_{\lambda \vee \mu}(a)_{\lambda \wedge \mu} \equiv(a)_{\lambda}(a)_{\mu}$ for all $\lambda, \mu \in \mathcal{P}_{n}$, so that (2.1) is satisfied as an equality. Hence, for $a_{1}, \ldots, a_{p}, b_{1}, \ldots, b_{q}>n-1$, the function

$$
\lambda \mapsto \frac{\left(a_{1}\right)_{\lambda} \cdots\left(a_{p}\right)_{\lambda}}{\left(b_{1}\right)_{\lambda} \cdots\left(b_{q}\right)_{\lambda}(n)_{\lambda}},
$$


$\lambda \in \mathcal{P}_{n}$, also is positive and $\mathrm{MTP}_{2}$.

Next, $s_{\lambda}(y)>0$ because $y \in \mathcal{H}_{n}^{+}$. Also, as we have observed in Section $2, s_{\lambda}(y)$ is $\mathrm{MTP}_{2}$ in $\lambda$. Again because the product of $\mathrm{MTP}_{2}$ functions remains $\mathrm{MTP}_{2}$, it follows that $c_{\lambda}$ ia positive and is $\mathrm{MTP}_{2}$ in $\lambda$.

Let us now draw from [5, 9, 15] the definition and some properties of the (generalized) hypergeometric functions of two Hermitian matrix arguments.

For each partition $\lambda=\left(\lambda_{1}, \ldots, \lambda_{n}\right) \in \mathcal{P}_{n}$, let $Z_{\lambda}$ denote the zonal polynomial indexed by $\lambda$. The zonal polynomials are spherical functions on $\mathcal{H}_{n}$ and, up to a constant multiple, $Z_{\lambda}$ coincides with the Schur functions: For $x \in \mathcal{H}_{n}$ with eigenvalues $x_{1}, \ldots, x_{n}$, the zonal polynomial is given by the explicit formula,

$$
Z_{\lambda}(x)=\omega_{\lambda} s_{\lambda}(x)
$$

where $s_{\lambda}(x) \equiv s_{\lambda}\left(x_{1}, \ldots, x_{n}\right)$ is given explicitly by the ratio formula (1.5), and

$$
\omega_{\lambda}=|\lambda| ! \frac{\prod_{j<k}\left(\lambda_{j}-\lambda_{k}-j+k\right)}{\prod_{j=1}^{n}\left(\lambda_{j}+n-j\right) !} .
$$

It is well-known, and follows as a limiting case of the formula (1.5), that $d_{\lambda}:=$ $s_{\lambda}(1, \ldots, 1)$ is given explicitly by the Weyl dimension formula,

$$
d_{\lambda}=\frac{\prod_{j<k}\left(\lambda_{j}-\lambda_{k}-j+k\right)}{\prod_{j=1}^{n}(j-1) !} .
$$

By (3.4),

$$
Z_{\lambda}\left(I_{n}\right)=\omega_{\lambda} d_{\lambda}
$$

and it can also be deduced from (3.5) and (3.6) that

$$
\omega_{\lambda}=\frac{|\lambda| ! d_{\lambda}}{(n)_{\lambda}}
$$

Although these results are well-known to have profound significance in the representation theory of $\operatorname{GL}(n, \mathbb{C})$, that subject will not play a role in our results.

Suppose that $a_{1}, \ldots, a_{p}, b_{1}, \ldots, b_{q} \in \mathbb{C}$ are such that, for $1 \leq i \leq q$ and $1 \leq$ $j \leq n$, none of the numbers $-b_{i}+j-1$ is a nonnegative integer.For $x, y \in \mathcal{H}_{n}$, the hypergeometric function of two Hermitian matrix arguments is defined as the series

$$
{ }_{p} F_{q}\left(\begin{array}{l}
a_{1}, \ldots, a_{p} \\
b_{1}, \ldots, b_{q}
\end{array} ; x, y\right)=\sum_{\lambda} \frac{\left(a_{1}\right)_{\lambda} \cdots\left(a_{p}\right)_{\lambda}}{\left(b_{1}\right)_{\lambda} \cdots\left(b_{q}\right)_{\lambda}} \frac{Z_{\lambda}(x) Z_{\lambda}(y)}{|\lambda| ! Z_{\lambda}\left(I_{n}\right)},
$$

where the sum is over all partitions $\lambda$ of all nonnegative integers.

For $x \in \mathcal{H}_{n}$, let $\|x\|=\max \left\{\left|x_{j}\right|: j=1, \ldots, n\right\}$ where $x_{1}, \ldots, x_{n}$ are the eigenvalues of $x$. Then the convergence properties of the series (3.9) are given in [5, Theorem 4.1]: If $p \leq q$ then the series (3.9) converges absolutely for all $x, y \in \mathcal{H}_{n}$; if $p=q+1$ then (3.9) converges absolutely if $\|x\| \cdot\|y\|<1$ and diverges if $\|x\| \cdot\|y\|>1$; and if $p>q+1$ then (3.9) diverges unless it terminates.

We can now establish a log-convexity property for the ${ }_{p} F_{q}$ functions in (3.9). 
Theorem 3.2. Let $x, y \in \mathcal{H}_{n}^{+}, p<q$, and $a_{1}, \ldots, a_{p+1}, b_{1}, \ldots, b_{q+1}>n-1$. Then,

$$
\begin{aligned}
{ }_{p+1} F_{q+1}\left(\begin{array}{c}
a_{1}, \ldots, a_{p+1} \\
b_{1}, \ldots, b_{q+1}
\end{array} ; x, y\right){ }_{p} F_{q}\left(\begin{array}{c}
a_{1}, \ldots, a_{p} \\
b_{1}, \ldots, b_{q}
\end{array} ;, y\right) \\
\leq{ }_{p+1} F_{q}\left(\begin{array}{c}
a_{1}, \ldots, a_{p+1} \\
b_{1}, \ldots, b_{q}
\end{array} ; x, y\right){ }_{p} F_{q+1}\left(\begin{array}{c}
a_{1}, \ldots, a_{p} \\
b_{1}, \ldots, b_{q+1}
\end{array} ; x, y\right) .
\end{aligned}
$$

If $p=q$ then the same result holds under the additional assumption that $\|x\| \cdot\|y\|<1$.

Proof. By (3.4), (3.7), and (3.8),

$$
\frac{Z_{\lambda}(x) Z_{\lambda}(y)}{|\lambda| ! Z_{\lambda}\left(I_{n}\right)}=\frac{s_{\lambda}(x) s_{\lambda}(y)}{(n)_{\lambda}}
$$

therefore, with the coefficients $c_{\lambda}$ as defined in (3.3), we have

$$
\sum_{\lambda \in \mathcal{P}_{n}} c_{\lambda} s_{\lambda}(x)={ }_{p} F_{q}\left(\begin{array}{l}
a_{1}, \ldots, a_{p} \\
b_{1}, \ldots, b_{q}
\end{array} ; x, y\right)
$$

For $a_{p+1}, b_{q+1}>n-1$, set $f(\lambda)=\left(a_{p+1}\right)_{\lambda}$ and $g(\lambda)=1 /\left(b_{q+1}\right)_{\lambda}$; then $f$ is increasing, and $g$ is decreasing, on $\mathcal{P}_{n}$. On applying the FKG inequality (3.1), we obtain

$$
\begin{aligned}
\left(\sum_{\lambda \in \mathcal{P}_{n}} c_{\lambda} s_{\lambda}(x) f(\lambda) g(\lambda)\right) \cdot\left(\sum_{\lambda \in \mathcal{P}_{n}} c_{\lambda} s_{\lambda}(x)\right) & \\
\leq & \leq\left(\sum_{\lambda \in \mathcal{P}_{n}} c_{\lambda} s_{\lambda}(x) f(\lambda)\right) \cdot\left(\sum_{\lambda \in \mathcal{P}_{n}} c_{\lambda} s_{\lambda}(x) g(\lambda)\right) .
\end{aligned}
$$

Substituting (3.3) for $c_{\lambda}$, we find that each sum reduces to a hypergeometric function of matrix argument, and then we obtain the desired result.

In [16, a class of generalizations of the FKG inequality with to up to five functions is provided. In the case of three functions, it is proved [16, Theorem 1.1] that if $\phi$ is a $\mathrm{MTP}_{2}$ density on $\mathbb{R}^{n}$ and $f, g$, and $h$ are nonnegative, increasing functions on $\mathbb{R}^{n}$ then

$$
2 \mathbb{E}(f g h)-[\mathbb{E}(f g) \mathbb{E}(h)+\mathbb{E}(f h) \mathbb{E}(g)+\mathbb{E}(f) \mathbb{E}(g h)]+\mathbb{E}(f) \mathbb{E}(g) \mathbb{E}(h) \geq 0,
$$

and analogous generalizations are given for four and five functions. By proceeding as before, choosing $c_{\lambda}$ as in (3.3), and choosing each of $f(\lambda), g(\lambda)$, and $h(\lambda)$ as a partitional shifted factorial or its inverse, we can obtain inequalities that extend Theorem 3.2

\section{References}

[1] N. Alon and J. H. Spencer, The Probabilistic Method, Third edition. Wiley, Hoboken, NJ, 2008.

[2] G. A. Battle and L. Rosen, "The FKG inequality for the Yukawa quantum field theory," J. Statist. Phys. 22 (1980), 123-192. 
[3] K. Fan, "An inequality for subadditive functions on a distributive lattice, with application to determinantal inequalities," Linear Algebra and Appl. 1 (1968), 3338.

[4] C. M. Fortuin, P. W. Kasteleyn, and J. Ginibre, "Correlation inequalities on some partially ordered sets," Comm. Math. Phys. 22 (1971), 89-103.

[5] K. I. Gross and D. St. P. Richards, "Total positivity, spherical series, and hypergeometric functions of matrix argument," J. Approx. Theory 59 (1987), 224-246.

[6] K. I. Gross and D. St. P. Richards, "Total positivity, finite reflection groups, and a formula of Harish-Chandra," J. Approx. Theory 82 (1995), 60-87.

[7] S. Helgason, Groups and Geometric Analysis, Academic Press, New York, 1984.

[8] I. Herbst and L. Pitt, "Diffusion equation techniques in stochastic monotonicity and positive correlations," Probab. Theor. Rel. Fields 87 (1991), 275 - 312.

[9] A. T. James, "Distributions of matrix variates and latent roots derived from normal samples," Ann. Math. Statist. 35 (1964), 475-501.

[10] S. Karlin, Total Positivity, Stanford University Press, Stanford, CA, 1968.

[11] S. Karlin and Y. Rinott, "Classes of orderings of measures and related correlation inequalities. I. Multivariate totally positive distributions," J. Multivariate Anal. 10 (1980), 467-498.

[12] T. Lam, A. Postnikov, and P. Pylyavskyy, "Schur positivity and Schur logconcavity," Amer. J. Math. 129 (2007), 1611-1622.

[13] T. Lam and P. Pylyavskyy, "Cell transfer and monomial positivity," J. Algebraic Combin. 26 (2007), 209-224.

[14] G. G. Lorentz, "An inequality for rearrangements," Amer. Math. Monthly 60, (1953), 176-179.

[15] I. G. Macdonald, Symmetric Functions and Hall Polynomials, Second edition. Oxford University Press, New York, 1995.

[16] D. St. P. Richards, "Algebraic methods toward higher-order probability inequalities, II," Ann. Probab., 32 (2004), 1509-1544.

[17] D. St. P. Richards, "Total positivity properties of generalized hypergeometric functions of matrix argument," J. Statist. Phys., 116 (2004), 225-231.

[18] R. P. Stanley, Enumerative Combinatorics, Volume 2. Cambridge University Press, Cambridge, 1999.

[19] D. M. Topkis, Supermodularity and Complementarity. Princeton University Press, Princeton, NJ, 1998. 